\title{
Nitric oxide contributes to induction of innate immune responses to gram-negative bacteria in Drosophila
}

\author{
Edan Foley and Patrick H. O'Farrell ${ }^{1}$ \\ Department of Biochemistry and Biophysics, University of California San Francisco, San Francisco, California 94143, USA
}

\begin{abstract}
Studies in mammals uncovered important signaling roles of nitric oxide (NO), and contributions to innate immunity. Suggestions of conservation led us to explore the involvement of NO in Drosophila innate immunity. Inhibition of nitric oxide synthase (NOS) increased larval sensitivity to gram-negative bacterial infection, and abrogated induction of the antimicrobial peptide Diptericin. NOS was up-regulated after infection. Antimicrobial peptide reporters revealed that NO triggered an immune response in uninfected larvae. NO induction of Diptericin reporters in the fat body required immune deficiency (imd) and domino. These findings show that NOS activity is required for a robust innate immune response to gram-negative bacteria, NOS is induced by infection, and NO is sufficient to trigger response in the absence of infection. We propose that NO mediates an early step of the signal transduction pathway, inducing the innate immune response upon natural infection with gram-negative bacteria.
\end{abstract}

[Keywords: Drosophila; nitric oxide; signaling; innate immunity; hemocyte; Relish]

Received June 25, 2002; revised version accepted November 4, 2002.

The innate immune system is an ancient first line of defense against foreign organisms (Hoffmann et al. 1999). In contrast to the genetic rearrangements and clonal selection processes that underlie adaptive immunity, innate immunity relies on the functions of germ-line encoded gene products. Nitric oxide (NO) is a highly reactive molecule with innate immune functions as well as roles in responses to hypoxia and CNS development (Dawson et al. 1991; Bredt and Snyder 1994; Gibbs and Truman 1998; Wingrove and O'Farrell 1999; Bogdan 2001). NO is produced in mammalian macrophages by a nitric oxide synthase isoform (iNOS/NOS2), which is strongly up-regulated following infection (MacMicking et al. 1997). Macrophages also produce high quantities of superoxide by NADPH oxidase following pathogen detection (Ding et al. 1988). The highly toxic molecules NO, superoxide, and their derivatives are believed to play an important role in destroying invading microorganisms (Nathan and Shiloh 2000). In seeking to develop Drosophila as a model for studying mechanisms modulating and directing this toxicity, we found that NO plays a signaling role in the induction of immune responses in Drosophila larvae to gram-negative bacteria.

Metazoan defenses against foreign organisms are commonly induced by pathogen detection. Components of

${ }^{1}$ Corresponding author.

E-MAIL ofarrell@cgl.ucsf.edu; FAX (415) 502-5145.

Article and publication are at http://www.genesdev.org/cgi/doi/10.1101/ gad.1018503. the innate immune system recognize chemical structures that are hallmarks of microorganisms and are not found on host cells (Akira et al. 2001). These pathogenassociated molecular patterns (PAMPs) include $\beta-1,3$ glucan of fungi, peptidoglycan and lipopolysaccharides of bacteria, and phosphoglycan of parasites. Widespread PAMPs are recognized by pattern recognition receptors (PRRs). PRR activation by binding of cognate PAMPs initiates host-signaling cascades that start defense responses.

Toll (Tl) signal transduction, which was originally identified on the basis of its role in dorsal/ventral patterning of the Drosophila embryo, is now recognized as having an evolutionarily conserved role in pathogen detection and induction of immune responses (Rosetto et al. 1995; Lemaitre et al. 1996; Nicolas et al. 1998; Qiu et al. 1998; Kopp and Medzhitov 1999; Aderem and Ulevitch 2000; Tauszig et al. 2000; Michel et al. 2001; Ooi et al. 2002; Tauszig-Delamasure et al. 2002; Underhill and Ozinsky 2002). The Drosophila Tl pathway is particularly responsive to fungi and gram-positive bacteria, which trigger processing and activation of the Tl ligand, Spaetzle (Spz; Levashina et al. 1999). Tl receptor-dependent signaling activates two NF- $\kappa$ B-related transcription factors, Dorsal (Dl) and Dorsal-related immunity factor (Dif), and expression of defense genes such as those encoding antimicrobial peptides (Lemaitre et al. 1995b; Meng et al. 1999).

In Drosophila, a pathway that is independent of $\mathrm{Tl}$, the Immune Deficiency (Imd) pathway, is activated in re- 
sponse to gram-negative bacteria. A putative peptidoglycan recognition protein (PGRP-LC) contributes to this pathway (Choe et al. 2002; Gottar et al. 2002; Ramet et al. 2002). Although there are uncertainties regarding its specificity, PGRP-LC has attributes of a PAMP and it acts upstream of the death-domain-containing protein Immune deficiency (Imd). Imd signaling proceeds through the MAP kinase kinase kinase homolog, dTAK1, the caspase, dredd, and an I-к-kinase to culminate in the proteolytic activation of the NF- $\kappa$ B family member Relish (Rel; Lemaitre et al. 1995a; Dushay et al. 1996; Wu and Anderson 1998; Hedengren et al. 1999; Kim et al. 2000; Silverman et al. 2000; Stoven et al. 2000; Georgel et al. 2001; Lu et al. 2001; Vidal et al. 2001). Mutants lacking Rel are highly sensitive to infection with gram-negative bacteria (Hedengren et al. 1999).

Two protocols have been established for investigating responses of Drosophila larvae to infection. Piercing the larval cuticle with a needle soiled with pathogen triggers a robust induction of the innate immune response. Whereas this septic injury protocol has been used successfully to define the outlines of the signaling cascades that induce host responses to infection, it has a disadvantage. The trauma of injury weakly activates immune responses even without bacteria, thus eroding the specificity of the response. A recently devised natural infection protocol activated the Imd pathway by briefly feeding larvae on concentrated slurries of a gram-negative bacteria such as Erwinia carotovora carotovora (Ecc; Basset et al. 2000).

We present evidence that NO makes crucial contributions to the Imd pathway that activates Rel. Pharmacological inhibition of NOS increases larval and adult susceptibility to septic and natural infection with gram- negative bacteria and compromises production of antimicrobial peptides. Treatment of uninfected larvae with NO donors is sufficient to activate antimicrobial peptide production. Genetic experiments demonstrate that one action of NO is either upstream of Imd or in a parallel pathway required for Rel activation of innate immune responses in the fat body. Ingestion of bacteria is followed by induction of NOS in the gut and in hemocytes. NO-mediated induction of the antimicrobial peptide Diptericin in the fat body was blocked in domino mutants. We propose that NO is an innate immune signal that acts early in a multi-tiered cascade in which sentinel tissues detect gram-negative bacteria and recruit other tissues to host defense.

\section{Results}

\section{NOS contributes to pathogen destruction}

We tested whether inhibition of NOS influences the ability of larvae to survive a bacterial infection. Drosophila has a single NOS homolog for which there are no available mutant alleles (Regulski and Tully 1995; Stasiv et al. 2001). To pharmacologically inactivate NOS, we treated larvae with the NOS-inhibitory arginine analog $\mathrm{N} \omega$-Nitro-L-Arginine-Methyl-Ester (L-NAME) and used the septic injury and natural infection models to infect third instar larvae with the Drosophila pathogen Ecc (Fig. 1A,B, respectively). In the absence of infection, treatment with L-NAME or the inactive D-enantiomer had little impact on eclosion/survival. Furthermore, injury alone did not significantly compromise eclosion/ survival. In contrast, L-NAME, but not D-NAME treatment dramatically reduced the ability of larvae to sur-
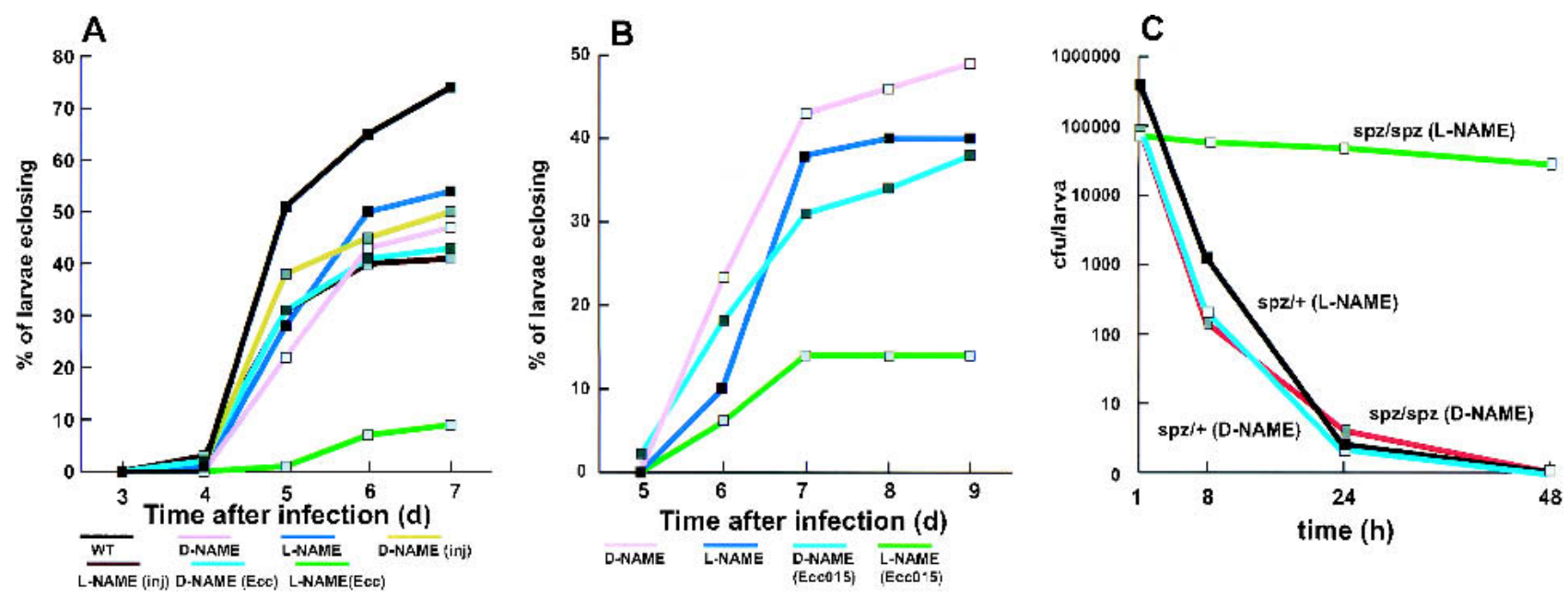

Figure 1. NOS activity contributes to pathogen destruction. $(A, B)$ The ability of third instar wild-type larvae to survive septic $(A)$ and natural $(B)$ infections and eclose. In both cases, inhibiting NOS by feeding L-NAME compromised the ability of the larvae to survive infection with Erwinia carotovora (L-NAME Ecc). In contrast, larvae survived infection when treated with a control isomer of the inhibitor (D-NAME Ecc) as well as enduring control treatments such as treatment with the drugs with or without injury (inj). (C) The bacterial titer at different times after Ecc015 natural infection of third instar larvae shows that host killing of bacteria is promoted by Spz-dependent and NOS-dependent processes. Whereas $s p z$ heterozygous larvae treated with L-NAME or D-NAME and $s p z$ mutants treated with D-NAME successfully destroyed invading bacteria, inactivation of Spz by mutation and NOS by L-NAME prevented host elimination of bacteria. 
vive a subsequent Ecc septic infection (Fig. 1A,B). Similar effects were observed with adult flies subjected to a septic infection (data not shown). We conclude that inactivation of NOS impairs the ability of Drosophila to combat subsequent infections by two different routes.

To distinguish contributions of NOS to the Imddependent and Spz-dependent defense pathways, we tested the consequence of NOS inhibition in mutant strains incapable of activating one or the other pathway. D-NAME had no effect and L-NAME only a modest effect on the ability of $s p z$ heterozygous larvae to eliminate bacteria (Fig. 1C; data not shown). In contrast, homozygous spz mutant larvae exhibited a remarkably different response to feeding D-NAME and L-NAME; at $24 \mathrm{~h}$ after infection, bacterial titer was 10,000 times higher in $s p z$ mutants fed L-NAME (Fig. 1C). This result indicates that L-NAME severely compromises Spz-independent host defenses. The major defense against gram-negative bacteria such as Ecc is thought to be mediated via the Spz-independent Imd pathway, which is thus a candidate target for the action of L-NAME.

Bacterial titer remained high in imd mutants whether or not they were fed L-NAME (data not shown). This is in accord with the possibility that L-NAME acts on the Imd pathway, but we cannot asses from this result whether NOS regulates any Imd-independent contributions to host defense, because such pathways do not ap- pear to confer any significant resistance to our infection protocol.

\section{NO is a signaling molecule in the Imd/Rel pathway}

The emphasized role of NO in the mammalian host defense is its involvement in pathogen killing. Studies in insect systems have also indicated a role for NOS in eliminating pathogens (Dimopoulos et al. 1998; Luckhart et al. 1998; Han et al. 2000), but there are suggestions that its action might include induction of immune responses (Nappi et al. 2000; Imamura et al. 2002). For this reason, we tested whether NO might have a role as an upstream regulator of the immune response by testing the influence of L-NAME treatment on induction of the antimicrobial peptide Diptericin (Dipt). Induction of Dipt in the fat body after a natural or septic infection is strictly dependent on Imd and Relish (Lemaitre et al. 1995a; Hedengren et al. 1999). We followed Dipt induction using a reporter construct in which GFP expression is controlled by the Dipt promoter (Dipt-GFP). This reporter mimics the infection-induced expression of the endogenous Dipt gene (Tzou et al. 2000).

No GFP was observed in Dipt-GFP larvae in the absence of infection. A large fraction $(79 \%)$ of larvae naturally infected with Ecc produced GFP in the fat body (Fig. $2 \mathrm{~B}, \mathrm{~F})$ within $16 \mathrm{~h}$. Whereas D-NAME did not compromise this induction (Fig. 2C,F), the fraction of GFP-expressing larvae was dramatically reduced by L-NAME
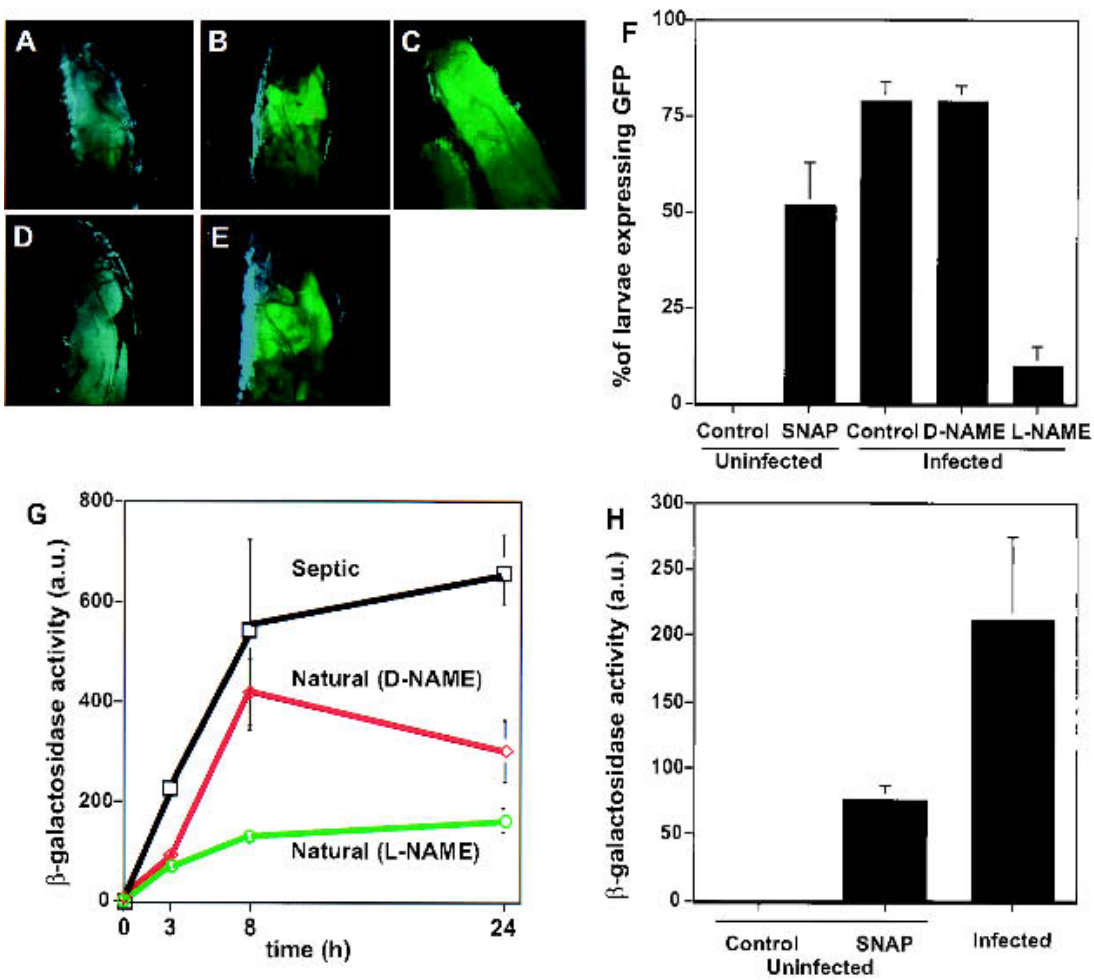

Figure 2. NO signaling activates expression of a Dipt reporter. $(A-E)$ GFP fluorescence in the anterior half of larvae that express GFP under the control of the Dipt promoter (DiptGFP). Uninfected larvae do not express the reporter $(A$, the weak green signal is background autofluorescence), whereas infection with Ecc induces reporter activity $(B)$. GFP expression persists in D-NAME-treated naturally infected larvae $(C)$, whereas it is absent in L-NAMEtreated naturally infected larvae $16 \mathrm{~h}$ postinfection $(D) .(E)$ The NO donor SNAP induces GFP in uninfected larvae. (F) Bar graph showing the percentage of larvae expressing GFP 16 $\mathrm{h}$ following the different treatments. $(G)$ Graph showing the $\beta$-galactosidase activity measured in larval extracts prepared from third instar larvae that express $\beta$-galactosidase under the control of the Dipt promoter (Dipt-lacZ) as a function of time after infection. Whereas DNAME-fed larvae display a robust $\beta$-galactosidase activity $8 \mathrm{~h}$ after natural infection, LNAME-fed larvae exhibit a lower $\beta$-galactosidase. The value for each time point is the average of three independent measurements The high levels of $\beta$-galactosidase activity in extracts from dipt-lacZ larvae exposed to a septic injury with Ecc015 persist somewhat longer than the levels in naturally infected larvae, but the initial responses differ only slightly. $(H)$ Graph showing $\beta$-galactosidase activity in Dipt-lacZ larvae prior to treatment with SNAP and $24 \mathrm{~h}$ after treatment. Provision of SNAP to uninfected larvae significantly increases $\beta$-galactosidase activity. Data are the average of three independent measurements. 
treatment (Fig. 2D,F, 10\%). Similar effects were seen after natural infection with Escherichia coli, although E. coli was not as potent as Ecc in inducing the reporter (data not shown). Thus, inhibition of NOS greatly reduced infection-dependent expression of the Dipt reporter.

To determine whether $\mathrm{NO}$ is sufficient to activate Dipt production on its own, uninfected Dipt-GFP larvae were fed the NO-donor S-Nitroso-N-acetylpenicillamine (SNAP) and examined $16 \mathrm{~h}$ later. Exposure to exogenous NO induced GFP in $~ 50 \%$ of the larvae despite the lack of infection (Fig. 2E,F). Control Dipt-GFP larvae treated with prereacted SNAP that no longer released NO did not express the reporter (data not shown). Therefore, we conclude that NOS activity is required for Dipt expression after natural infection with gram-negative bacteria, and that NO is sufficient to activate Dipt expression in the absence of infection. Both infection and SNAP treatment resulted in an initial mosaic pattern of GFP in the fat body (data not shown; Reichhart et al. 1992). Thus, exogenously provided NO not only activates the Imd pathway in the absence of infection, it also recapitulates this spatial feature of Dipt expression.

To compare the efficacy of natural and septic infections, and to assess the degree of inhibition of the response by L-NAME, we measured $\beta$-galactosidase activity in extracts of treated larvae that express $\beta$-galactosidase under the control of the Diptericin promoter (Dipt-lacZ). Septic infection produced a slightly faster and more persistent rise in $\beta$-galactosidase than natural infection, but this difference was small compared with the suppression of the response upon incubation with L-NAME (Fig. 2G). Note that there is a slow rise in $\beta$-galactosidase even in the presence of L-NAME, and that control larvae appear to be down-regulating the response within $24 \mathrm{~h}$ (Fig. 2G). We also quantified the $\beta$-galactosidase activity of uninfected, SNAP-treated Dipt-lacZ larvae $24 \mathrm{~h}$ after feeding SNAP. Exposure to NO led to a clear elevation of $\beta$-galactosidase activity in uninfected larvae (Fig. 2H), supporting the results with Dipt-GFP (Fig. 2E,F), indicating that $\mathrm{NO}$ is sufficient to induce Dipt expression in uninfected larvae.

In summary, pharmacological inhibition of NOS inhibits, or greatly retards induction of Dipt reporter constructs in response to infection, whereas dietary provision of NO to larvae stimulates Dipt production in the absence of infection. We propose that NO contributes to the activation of the Imd/Rel pathway. As L-NAMEtreated wild-type larvae eliminate pathogens more effectively than imd mutants, we believe that pharmacological inhibition of NOS does not completely inactivate Imd-dependent signaling. In agreement with this, we noticed that a low percentage of L-NAME treated infected Dipt-GFP larvae still express GFP upon infection and that, although greatly reduced and delayed, low-level reporter induction occurred in the presence of L-NAME. Nonetheless, the contribution of NOS to gram-negative bacterial destruction is substantial, as L-NAME treated $s p z$ mutants are greatly impaired in their ability to clear Ecc upon natural infection when compared with untreated control spz mutant larvae.

\section{NO effects on Drosomycin expression}

Whereas Dipt is induced strictly via the Imd pathway, Drosomycin (Drs) is also induced by the Tl pathway (Lemaitre et al. 1996). To test whether NOS is required for this response, we examined induction of a reporter expressing GFP under the control of the Drs promoter (Drs-GFP). A few uninfected larvae (7\%) exhibited foci of Drs-GFP expression, but natural infection with Ecc dramatically increased the incidence and extent of GFP expression $16 \mathrm{~h}$ post infection (Fig. 3B). The number of larvae exhibiting this infection-induced Drs expression was not appreciably affected by D-NAME or L-NAME treatment ( $88 \%$ and $80 \%$, respectively; Fig. 3F). Thus, it appears that inhibition of NOS does not prevent infection-dependent Drs induction. Nonetheless, the NO donor SNAP induced GFP in $~ 50 \%$ of uninfected Drs-GFP larvae (Fig. 3E,F).

To quantify contributions of NO to Drs expression, we prepared extracts from naturally infected larvae that express $\beta$-galactosidase under control of the Drs promoter (Drs-1acZ). Following infection, L-NAME fed larvae had significantly less (by nearly half) $\beta$-galactosidase activity than D-NAME fed larvae (Fig. 3G). Thus, it appears that although NOS is not required for all Drs reporter activity, NOS activity makes a quantitative contribution. Consistent with a quantitative contribution of $\mathrm{NO}$ to the induction, we observed that SNAP mediated a substantial induction of Drs-lacZ in uninfected larvae (Fig. $3 \mathrm{H})$. Whereas the most straightforward interpretation of these results is that infection-mediated induction of Drs occurs via two pathways (see Discussion), a NOS dependent and independent one, we cannot exclude the possibility that L-NAME only partially blocks a single NOdependent pathway.

\section{Epistatic relations of NOS and Imd in the Imd/Rel pathway}

To test whether NO induction of a Dipt reporter involves the Imd pathway, we tested the response in the fat bodies of imd mutant larvae. As shown by activity stain for $\beta$-galactosidase in dissected fat bodies from DiptlacZ larvae (Fig. 4), NO induction of the reporter requires Imd function. Reciprocally, overexpression of Imd, which activates immune responses (Georgel et al. 2001) and reporter expression (Fig. 4H) in the absence of infection, retained its ability to activate Dipt-lacZ expression in the presence of the NOS inhibitor, L-NAME (Fig. 4I). These findings are most simply consistent with action of NO upstream of Imd in the signal transduction pathway inducing Dipt in the fat body, although it remains possible that NO acts in a parallel pathway.

\section{NOS involvement in hemocyte responses to infection}

In addition to the fat body, other cells such as hemocytes produce antimicrobial peptides after infection. To test whether NO impinges upon hemocyte expression of antimicrobial peptides, we naturally infected or SNAP 

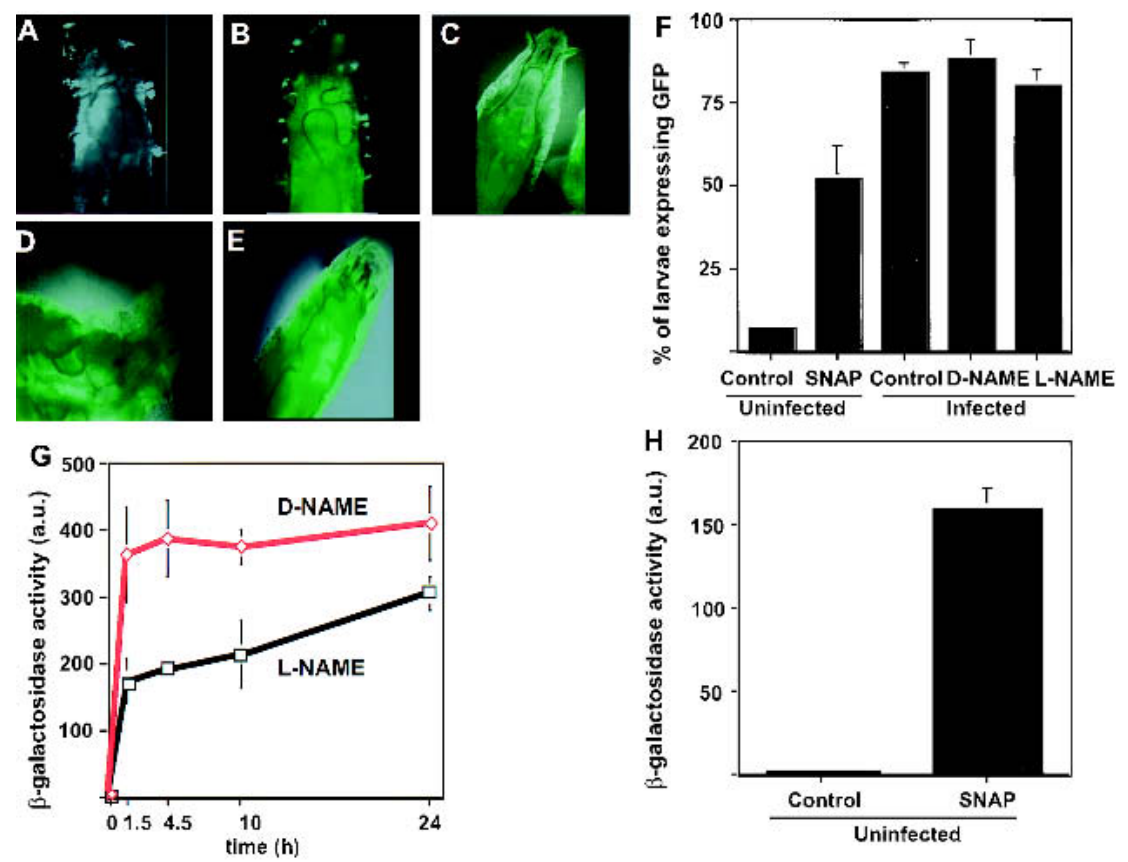

Figure 3. NO activates expression of a Drs reporter, but NOS inhibition only partially reduces Drs induction after infection. $(A-E)$ GFP fluorescence in the anterior half of larvae that express GFP under the control of the Drs promoter (Drs-GFP). Most uninfected larvae do not express the reporter $(A)$, whereas larvae naturally infected with $E c c$ do $(B)$. Naturally infected larvae treated with either D-NAME $(C)$ or L-NAME $(D)$ produce GFP, and provision of SNAP to uninfected larvae induces GFP $(E) .(F)$ Bar graph showing the percentage of larvae expressing GFP following the different treatments. $(G)$ Graph showing the $\beta$-galactosidase activity measured in larval extracts prepared at different times after natural infection from third instar larvae that express $\beta$-galactosidase under the control of the Drs promoter (Drs-lacZ). $\beta$-galactosidase activity in L-NAME-fed, infected larvae are consistently half of those in D-NAME-fed, infected larvae. The value for each time point is the average of three independent measurements. $(H)$ Graph showing $\beta$-galactosidase activity in Drs-lacZ larvae prior to treatment with
ignificantly increases $\beta$-galactosidase activity.

SNAP and $24 \mathrm{~h}$ after treatment with SNAP. Provision of SNAP to uninfected larvae significantly increases $\beta$-galactosidase activity. Data are the average of three independent measurements.

treated Dipt-lacZ larvae, collected hemocytes $16 \mathrm{~h}$ later, and stained for $\beta$-galactosidase activity. We did not observe Dipt-lac $Z$ activity in hemocytes from uninfected larvae (Fig. 5A), whereas a subset of hemocytes from larvae infected with $E c c$ had deposits of X-gal derived stain (Fig. 5B). This induction was also observed following treatment of uninfected larvae with SNAP (Fig. 5C). Infection-dependent induction of Dipt was blocked by LNAME (Fig. 5D). We conclude that NO is necessary for efficient induction of Dipt in larval hemocytes following natural infection.

In mammalian systems, one of three NOS isoforms, inducible NOS (iNOS/NOS2), is up-regulated in macrophages in response to several types of infection or challenge with bacterial lipopolysaccharides. We asked whether infection up-regulates NOS in Drosophila hemocytes, the majority of which are phagocytic cells that function like mammalian macrophages. Larvae were infected by feeding with Ecc, and hemocytes were collected after $5 \mathrm{~h}$ and examined for NOS protein levels by immunofluorescence. We used two different anti-NOS antibodies; a universal anti-NOS and an antibody we prepared against a C-terminal peptide of Drosophila NOS. Similar results were observed for both antibodies. Hemocytes of infected larvae showed elevated NOS protein levels as early as $5 \mathrm{~h}$ after infection (Fig. $5 \mathrm{H}$ ).

Because host pathogen interaction following natural infection is likely to first occur in the gut, we considered whether there might also be a response in this tissue. We monitored NOS activity in the guts of infected larvae using a histochemical stain (diaphorase staining). We observed a striking increase in NOS activity levels in the guts of naturally infected larvae $5 \mathrm{~h}$ after infection with Ecc (Fig. 5L).
NO-dependent activation of Dipt production in fat bodies requires the function of domino

Mutations in the chromatin-remodeling factor, domino, affect proliferating tissues, including imaginal tissues and larval hemocytes, and result in pupal lethality (Ruhf et al. 2001). It has been demonstrated previously that domino mutants are incapable of producing Dipt in their fat body after natural infection with Ecc, whereas fat body production of Drs was not impaired (Basset et al. 2000). This result has implicated hemocytes, which are missing in domino mutants, in the response to bacteria; however, as the mutation is relatively pleiotropic, other explanations for the domino dependence are possible. Nonetheless, we tested whether the domino mutation similarly impairs SNAP-mediated induction of Drs-GFP or Dipt-GFP.

Uninfected Drs-GFP larvae occasionally express GFP (Fig. 3F) and a low level of background expression persisted in domino mutants (Fig. 6A). As described previously, Drs-GFP; domino mutants expressed GFP after natural infection with Ecc (Fig. 6C). Interestingly, uninfected domino mutant larvae also induced Drs-GFP after feeding SNAP (Fig. 6D). Thus, Drs reporter induction in response to exposure to an NO donor or to bacteria can occur in the absence of hemocytes. Thus, cells other than, or in addition to, hemocytes must respond to NO and have inputs into the immune response.

We observed different effects when we examined domino; Dipt-GFP larvae. In this case, we observed no GFP fluorescence after either natural infection with Ecc or treatment with SNAP (Fig. 6F, G, respectively). These data show a parallel between NO signaling and the Imd pathway, in that both are blocked in the domino mutant. 


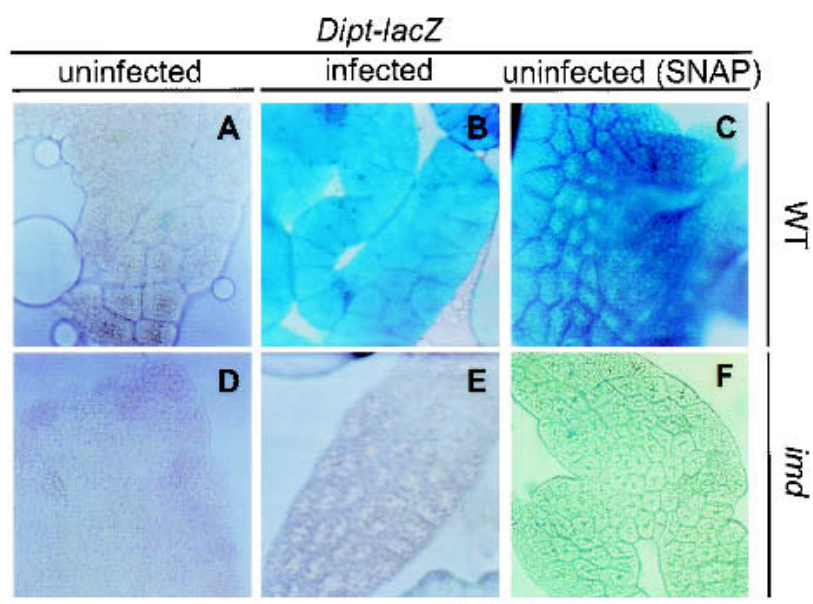

Dipt-lacZ; hsGAL4/UASimd

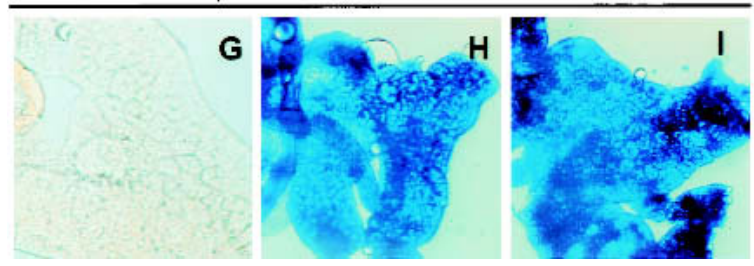

Figure 4. NO action in the fat body requires Imd. $(A-I)$ Dissected fat bodies stained for $\beta$-galactosidase activity derived from a Dipt-lacZ reporter transgene. $(A-C)$ In wild-type larvae carrying the dipt-lac $Z$ transgene, natural infection $(B)$ or exposure of uninfected larvae $(C)$ to the NO donor SNAP induces Dipt-lacZ expression within $24 \mathrm{~h}$ (see Fig. $2 \mathrm{H}$ for measurement of induction levels). In contrast, no staining was observed in the fat bodies of imd mutant larvae $24 \mathrm{~h}$ after exposure to bacteria $(E)$ or to SNAP $(F)$. hsGAL4 and UAS-imd transgenes allowed indirect induction of Imd expression in response to heat shock. The fat bodies of dipt-lacZ; hsGAL4/UAS-imd larvae showed no detectable lac $Z$ staining in the absence of a heat shock $(G)$, but staining was readily evident $24 \mathrm{~h}$ after administration of a heat shock, irrespective of whether the larvae had been larvae raised on D-NAME- $(H)$ or L-NAME- $(I)$ containing fly food.

Furthermore, this finding suggests that the fat body does not have an autonomous ability to respond to $\mathrm{NO}$, and that the NO role in the induction of Dipt-GFP occurs at stages of signaling upstream of the fat body. Finally, the distinction between the effect of the domino mutation on Drs and Dipt reporters demonstrates that NO stimulates Drs expression via a pathway that is, at least partially, distinct from the pathway by which NO induces Dipt. We speculate that both pathways involve distinct sentinel tissues that act upstream of the fat body (Fig. 7).

\section{Discussion}

Drosophila has emerged as a potent model for elucidation of mechanisms underlying innate immunity. Our data show that NO performs a signaling function in Drosophila in the induction of host defenses to gram-negative bacteria. Pharmacological inactivation of Drosophila NOS makes larvae more susceptible to infection and blocks Rel-dependent activation of the antimicrobial peptide Diptericin. NOS is up-regulated in the gut and hemocytes following natural infection, consistent with a role for NOS in combating invading micro-organisms. Furthermore, dietary provision of an NO donor is sufficient to activate promoters of antimicrobial genes in the hemocytes and fat body of uninfected hosts. Tests of the ability of NO to induce fat body expression of Diptericin in mutant backgrounds showed that it cannot bypass the requirement for Imd, a component of the signal transduction pathway inducing Dipt.

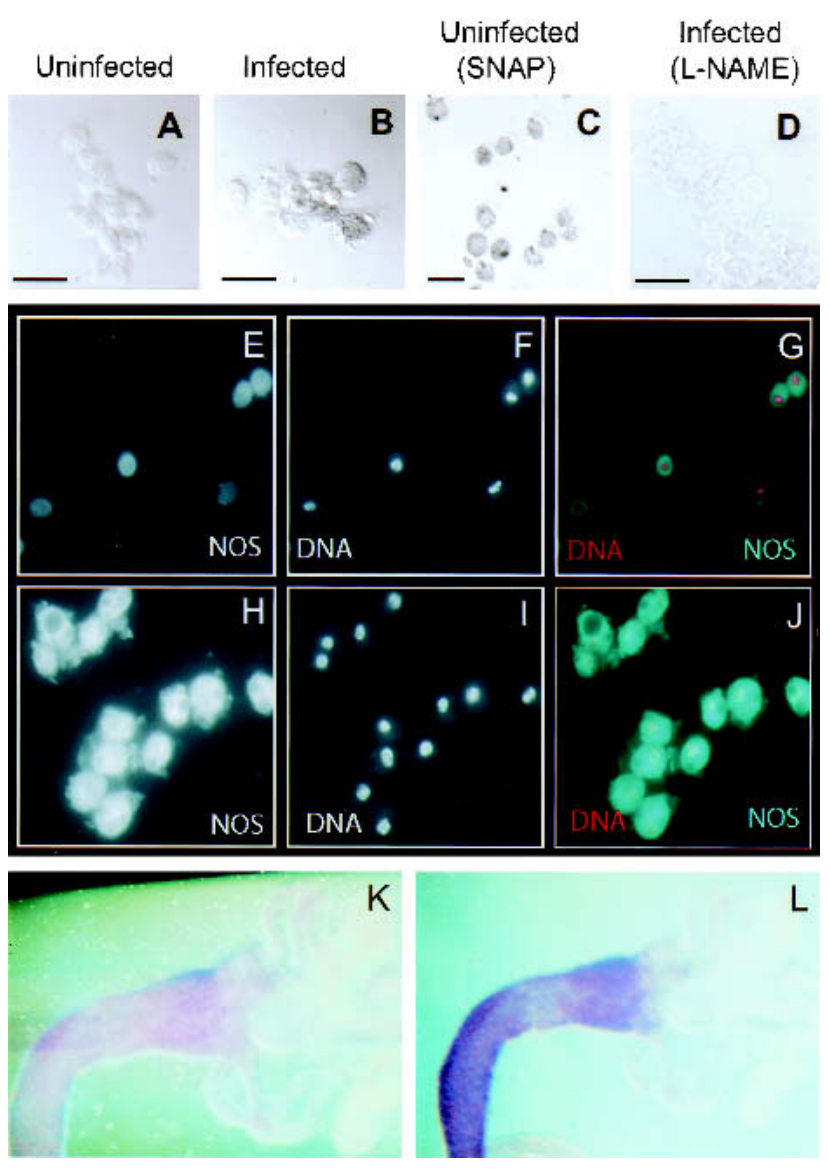

Figure 5. Expression of Dipt-lacZ in hemocytes requires NOS. $(A-D) \beta$-galactosidase activity in hemocytes from Dipt-lac Z larvae. Hemocytes from uninfected larvae have no $\beta$-galactosidase activity $(A)$, whereas a subset of hemocytes from naturally infected larvae show staining $(B)$. Provision of SNAP to uninfected larvae induces $\beta$-galactosidase $(C)$, whereas L-NAME treatment of larvae inhibits induction upon natural infection $(D)$. (E-J) Immunohistochemical staining showing NOS protein levels and Hoechst staining showing DNA in hemocytes from uninfected $(E-G)$ and infected $(H-J)$ larvae. $E$ and $F$ were merged in $G$, and $H$ and $I$ were merged in $J$ with NOS in blue and DNA in red. The images in $E$ and $H$ were taken at the same magnification with identical exposure times. $(K-L)$ The diaphorase staining of guts dissected from uninfected and naturally infected larvae. The diaphorase staining is significantly lower in the guts of uninfected larvae $(K)$ compared with naturally infected larvae (L). Bar, $20 \mu \mathrm{m}$. 

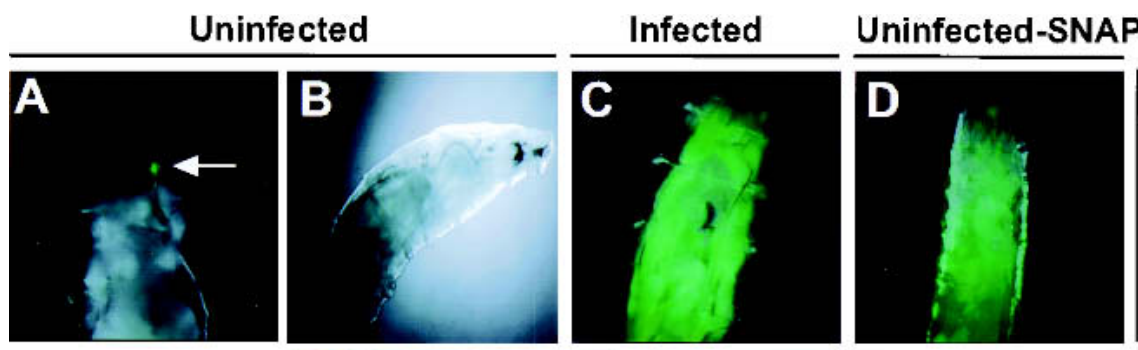

Drs-GFP; domino
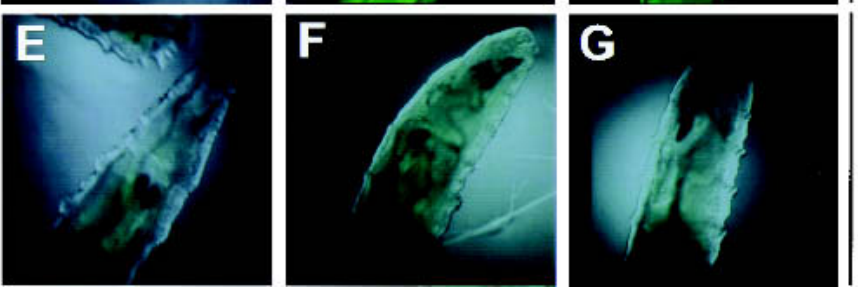

domino;

Dipt-GFP

Figure 6. The domino mutant interferes with NO induction of Dipt, but not induction of Drs. $(A-D)$ Expression of a Drs-GFP reporter transgene in the anterior of domino mutant third instar larvae. Localized GFP fluorescence is seen in a subset of uninfected larvae $(A$, arrow), whereas the majority of uninfected larvae do not exhibit detectable fluorescence $(B)$. Natural infection $(C)$ or provision of SNAP $(D)$ to uninfected larvae induces the Drs reporter. $(E-G)$ Fluorescence images of domino mutant third instar larvae carrying the Dipt-GFP transgene show that neither bacterial feeding $(F)$ nor exposure to SNAP $(G)$ induce expression of the Dipt reporter.

\section{Inhibition of NOS impairs the host defense response}

Our initial experiments showed that pharmacological inactivation of NOS compromised the ability of Drosophila larvae or adults to survive infection by septic injury or natural infection. Furthermore, NOS inhibition by L-NAME limited the ability of naturally infected larvae to eliminate gram-negative bacteria (Ecc and E. coli). L-NAME attenuation of pathogen elimination was particularly dramatic in larvae incapable of mounting Spzdependent responses to infection. In contrast, an imd mutant was not further compromised by NOS inhibition. These results suggest that NOS activity is of particular importance in the Imd-dependent immune response pathway.

Production of Dipt in the fat body following infection relies entirely on the Imd-signaling cascade, as mutations in imd or the Imd-responsive NF-кB homolog rel completely abrogate Dipt production following infection (Lemaitre et al. 1995a; Hedengren et al. 1999; Georgel et al. 2001). Overproduction of Imd in the absence of infection activates Dipt production (Georgel et al. 2001). Thus, the Imd pathway is required for Dipt activation and is sufficient to induce Dipt expression. Similarly, we show that pharmacological inhibition of NOS reduces Dipt expression, whereas introduction of NO donors activates Dipt expression. Thus, NO is required for optimal Dipt induction and NO is sufficient to trigger Dipt expression.

We propose that NO has a signaling role in the innate immune system of Drosophila and contributes to pathogen resistance in this capacity (Fig. 7). We note that this role in host defense is distinct from roles that NO might play as a cytotoxic agent used in the destruction of pathogens. Previous studies have indicated signaling functions for NO in the immune response of mammals and plants (Delledonne et al. 1998; Durner et al. 1998;
Diefenbach et al. 1999) although the exact manner in which NO functions has not been elucidated. Our data demonstrate that NO is essential for activation of a specific branch of the innate immune response pathway; the Imd pathway in Drosophila that is analogous to the TNF pathway of mammals.

\section{Interactions between NO and the T1-dependent response pathway}

Our data imply a crucial role for NO in Imd-dependent signaling. However, NO also can activate production of Imd-independent antimicrobial peptides, as dietary provision of NO is sufficient to activate Drs production. Although this finding does not necessarily imply that NO normally acts in this pathway, it is of interest to consider the relationship to the results we have obtained for NO induction of the Imd pathway.

There is some cross-talk between the Toll and Imd pathways, such that activation of the Imd pathway by NO might contribute to the induction of Drs. However, the Imd pathway is not necessary for induction of Drs by exogenous NO, as a dietary NO source induces Drs to an equal extent in wild-type, imd and $r e{ }^{E 2 O}$ mutants (data not shown). Hence, there must be another route of activation of Drs by exogenous NO. However, as inhibition of NOS does not effectively inhibit the Tl pathway as it does the Imd pathway, further analyses will be required to determine whether NO normally makes contributions to the Tl-dependent innate immune responses. Here, we focus on NO contributions to the Imd pathway.

The domino mutation and NO-dependent activation of antimicrobial peptide production

The Domino gene product is a transcription factor involved in chromatin remodeling (Ruhf et al. 2001). Mu- 


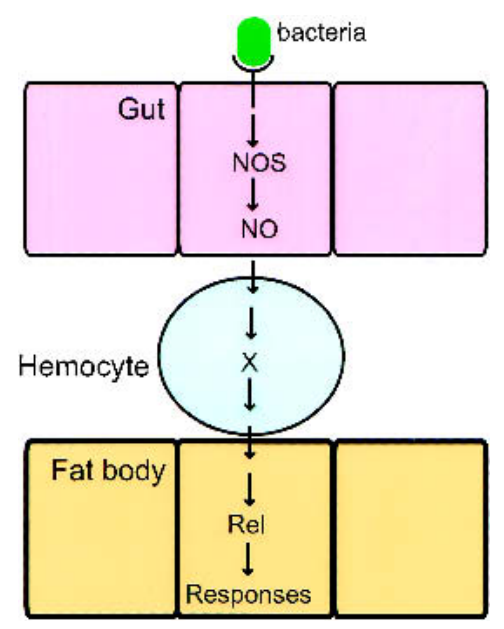

Figure 7. A model for NO signaling in immunity after natural infection of Drosophila larvae. Receptors, the PRRs on the surface of sentinel tissues, recognize molecules peculiar to pathogens. We suggest that the gut serves such a sentinel function in the case of natural infection, but note that other tissues such as hemocytes might adopt this role upon systemic infection. We propose that host-pathogen interactions up-regulate NOS in sentinel tissues (Fig. 5; Han et al. 2000) and activate a signaling cascade that leads to the release of a cytokine-like activity that recruits additional tissues to defend against the pathogen. We propose that hemocytes function as an intermediary in the Imd pathway that culminates in fat body production of antimicrobial peptides. It should be noted that the classical genes of the Imd pathway might act in different tissues or might act repeatedly at more than one tier of the cascade. As the septic injury model circumvents the gut as a point of entry for pathogens, it is likely that other tissues will act as sentinels mediating the response or the fat body might respond directly under these circumstances.

tations in domino affect proliferating tissues such as the imaginal discs and lymph glands, resulting in larvae with several defects, including elimination or severe reduction in the number of hemocytes (Braun et al. 1998). Whereas domino mutants produce Dipt in the proventriculus after natural infection, they no longer produce it in the fat body. These observations led to the proposal that hemocytes may produce a cytokine-like molecule following natural infection that activates antimicrobial peptide production in the fat body (Basset et al. 2000). Whereas the proposal that the fat body response is secondary to a hemocyte signal remains tentative because of the pleiotropy of the domino mutation, it is clear that a gut (proventriculus) response can occur independent of hemocytes. We find that NO also fails to induce Dipt in the fat body of domino mutant larvae, suggesting that NO does not act directly on the fat body. Perhaps it mediates signaling at earlier steps in the response.

We propose that different host organs play distinct roles in the response to foreign organisms. Tissues that contact a pathogen early during infection are well positioned to perform a sentinel function by detecting the pathogen and signaling other tissues to recruit their contributions to host defense (Fig. 7). Our results are consis- tent with the suggested involvement of hemocytes in the response of the fat body (Basset et al. 2000). The finding that exogenous NO is unable to induce Dipt in the fat body of domino mutants, which lack hemocytes, suggests that the fat body cannot respond autonomously to NO. Although the pleiotropy of the mutation allows other interpretations, the result encourages us to think that NO acts upstream of the fat body. In support of this, the initial fat body response to NO or infection appears as a mosaic of Dipt expressing and nonexpressing cells. This feature is not easily consistent with a humoral signal, but is compatible with a induction by cell contact with activated hemocytes. Furthermore, hemocytes respond to natural infection by inducing Dipt. Because this response is mimicked by NO treatment and inhibited by pharmacological inhibition of NOS, we suggest that NO acts upstream of this hemocyte response. It was shown previously that the gut (proventriculus) can respond to infection independent of, and perhaps upstream of hemocytes (Basset et al. 2000). We show that NOS activity is induced in the gut following natural infection. These findings suggest the tentative model shown in Figure 7, in which cells in the gut detect the pathogen, activate hemocytes via an NO dependent signal, and hemocytes act in turn to induce the fat body by an as yet unknown signal. As pathogens have numerous ports of access, we expect that, like the diverse dendritic system in humans, there will be a number of alternative sentinel tissues and multiple pathways of triggering activation of innate immunity.

Our work and other studies indicate that NO has a global involvement in the immune response. Work in mammalian cells predicted involvement of NO in pathogen elimination during an oxidative burst in phagocytic cells, whereas our work documents a role for NO as an inducer of the innate immune response. It remains to be tested fully, but several observations suggest that both of these roles will be conserved.

\section{Materials and methods}

\section{Fly strains and culturing}

We used Sevelen flies as a wild-type strain. The $s p z^{r m 7}$, domino, and imd mutant strains used have been described elsewhere (Lemaitre et al. 1995a, 1996; Braun et al. 1998; Georgel et al. 2001). The reporter strains Dipt-lacZ, Drs-GFP, and Drs-lacZ have been described in Reichhart et al. (1992), Ferrandon et al. (1998), and Manfruelli et al. (1999), respectively. Dipt-GFP flies were a gift of Bruno Lemaitre. It is a homozygous viable strain with two insertions on each third chromosome. In this line, GFP is expressed under the control of the Dipt 2.2-kb promoter, which recapitulates in vivo Dipt expression. Flies were cultured on standard fly food at $25^{\circ} \mathrm{C}$ prior to infection. After infection, flies were transferred to a $29^{\circ} \mathrm{C}$ incubator.

\section{Infection experiments}

Natural infection experiments with $E c c$ or E. coli were done as described previously (Basset et al. 2000). Briefly, for $30 \mathrm{~min}$, third instar larvae were fed a concentrated bacterial pellet 
mixed into crushed banana with $500 \mathrm{mM}$ L-NAME or D-NAME. Larvae were then transferred to $29^{\circ} \mathrm{C}$ for the remainder of the experiment. To determine the consequences of NOS inhibition on larval survival after infection, larvae were transferred to vials that contained instant fly food with $100 \mathrm{mM}$ L-NAME or D-NAME, respectively. Bacterial titer was determined by plating serial dilutions of homogenate from infected larvae at various times after infection onto LB-Amp plates. The strain of Ecc used (015) is ampicillin resistant and E. coli was transfected with a $\beta$-lactamase encoding plasmid. For septic injury experiments on larvae, late second instar larvae were transferred from standard fly food to instant fly food containing 100 mM D-NAME or L-NAME. A total of 120 third instar larvae were picked $16 \mathrm{~h}$ later and infected by puncturing the cuticle with a dissecting needle that had been soaked previously in a concentrated bacterial pellet. In parallel control experiments, 120 larvae were treated in an identical manner and injured with a sterile needle. For septic injury of adults, late second instar larvae were transferred from standard cornmeal food to instant fly food with $100 \mathrm{mM}$ L-NAME or D-NAME. Two-hundred 1 to 2-day-old adult flies that eclosed were infected by puncturing their thorax with a sharp needle that had been dipped in a concentrated bacterial pellet.

\section{Microscopy}

All images were taken on a Leica DMRD. To visualize expression of GFP in larvae, darkfield and green fluorescent images (FITC) were taken of representative larvae and merged using Adobe Photoshop 5.5. Figures were assembled using Adobe Illustrator. For immunohistochemistry of larval hemocytes, hemocytes were deposited on Superfrost Plus Gold microscope slides (Fisher) and fixed for $5 \mathrm{~min}$ in $4 \%$ glutaraldehyde in PBS. Cells were incubated with a 1:200 dilution of Rabbit-anti-NOS and Rhodamine-labeled anti-rabbit was used a secondary antibody. NOS antibody staining was done using a universal antiNOS antibody (Oncogene) or using an antibody raised against a peptide (TAEIHTKSRATARIRMASQ) that corresponds to a Cterminal section of the mature NOS protein.

\section{$\beta$-Galactosidase assays}

To monitor induction of Dipt-lacZ in fat bodies, fat bodies were dissected from larvae in PBS and fixed for 10 min in PBS with $0.5 \%$ glutaraldehyde on ice. Fat bodies were then incubated at room temperature in staining buffer $[30 \mu \mathrm{L} \mathrm{X-gal} \mathrm{(5 \%} \mathrm{5-bromo-}$ 4-chloro-3-indolyl $\beta$-D-galactosidase in DMFO/ per milliliter staining solution $(10 \mathrm{~m}$ of sodium phosphate buffer at $\mathrm{pH} 7.2$, $150 \mathrm{mM} \mathrm{NaCl}, 1 \mathrm{mM} \mathrm{MgCl}, 3.5 \mathrm{mM} \mathrm{K} 3 \mathrm{FeCN} 6$, and $3.5 \mathrm{mM}$ K4FeCN6)]. $\beta$-galactosidase activity in hemocytes from Diptlac $Z$ larvae was monitored by depositing hemolymph on Superfrost Plus Gold microscope slide (Fisher), fixing for $30 \mathrm{sec}$ in PBS with $0.5 \%$ glutaraldehyde, and staining overnight at $37^{\circ} \mathrm{C}$ with staining buffer. $\beta$-galactosidase titration was done as described previously (Basset et al. 2000).

\section{Diaphorase staining}

Third instar larvae were washed and dissected in PBS and then transferred to fixing buffer $(4 \%$ paraformaldehyde, $100 \mathrm{mM}$ PIPES at pH 7.4, $2 \mathrm{mM} \mathrm{MgSO}_{4}, 1 \mathrm{mM}$ EGTA) for $30 \mathrm{~min}$ at room temperature. Larvae were then washed in PBS and incubated in staining solution $(1 \mathrm{mM} \mathrm{NADPH}, 0.2 \mathrm{mM}$ nitrobluetetrazolium, $100 \mathrm{mM}$ Tris at $\mathrm{pH}$ 7.2, 0.2\% Triton X-100) for $20 \mathrm{~min}$ at room temperature. Larvae were washed in PBS and mounted in glycerol.

\section{Pharmacology}

The NO donor SNAP was fed to third instar larvae for $15 \mathrm{~min}$ at room temperature by mixing $15 \mathrm{mM}$ SNAP in crushed banana and then feeding to larvae.

\section{Acknowledgments}

Fly strains were kindly provided by Kathryn Anderson, Jules Hoffmann, Bruno Lemaitre, and Marie Meister. We thank Bruno Lemaitre for advice and discussions. We also thank Pascale Dijkers, Arnaud Echard, Renny Feldman, Gilles Hickson, Devin Parry, and Anita Sil for critical reading of the manuscript. This work was supported by a basic Science Award from the Sandler Family Supporting Foundation and N.I.H. grant GM60988. Supported in part by Fellowship DRG-1713-02 from the Damon Runyon Cancer Research Foundation.

The publication costs of this article were defrayed in part by payment of page charges. This article must therefore be hereby marked "advertisement" in accordance with 18 USC section 1734 solely to indicate this fact.

\section{References}

Aderem, A. and Ulevitch, R.J. 2000. Toll-like receptors in the induction of the innate immune response. Nature 406: 782787.

Akira, S., Takeda, K., and Kaisho, T. 2001. Toll-like receptors: Critical proteins linking innate and acquired immunity. Nat. Immunol. 2: 675-680.

Basset, A., Khush, R.S., Braun, A., Gardan, L., Boccard, F., Hoffmann, J.A., and Lemaitre, B. 2000. The phytopathogenic bacteria Erwinia carotovora infects Drosophila and activates an immune response. Proc. Natl. Acad. Sci. 97: 3376-3381.

Bogdan, C. 2001. Nitric oxide and the immune response. Nat. Immunol. 2: 907-916.

Braun, A., Hoffmann, J.A., and Meister, M. 1998. Analysis of the Drosophila host defense in domino mutant larvae, which are devoid of hemocytes. Proc. Natl. Acad. Sci. 95: 1433714342.

Bredt, D.S. and Snyder, S.H. 1994. Nitric oxide: A physiologic messenger molecule. Annu. Rev. Biochem. 63: 175-195.

Choe, K.M., Werner, T., Stoven, S., Hultmark, D., and Anderson, K.V. 2002. Requirement for a peptidoglycan recognition protein (PGRP) in Relish activation and antibacterial immune responses in Drosophila. Science 296: 359-362.

Dawson, T.M., Bredt, D.S., Fotuhi, M., Hwang, P.M., and Snyder, S.H. 1991. Nitric oxide synthase and neuronal NADPH diaphorase are identical in brain and peripheral tissues. Proc. Nat1. Acad. Sci. 88: 7797-7801.

Delledonne, M., Xia, Y., Dixon, R.A., and Lamb, C. 1998. Nitric oxide functions as a signal in plant disease resistance. $\mathrm{Na}$ ture 394: 585-588.

Diefenbach, A., Schindler, H., Rollinghoff, M., Yokoyama, W.M., and Bogdan, C. 1999. Requirement for type 2 NO synthase for IL-12 signaling in innate immunity. Science 284: 951-955.

Dimopoulos, G., Seeley, D., Wolf, A., and Kafatos, F.C. 1998. Malaria infection of the mosquito Anopheles gambiae activates immune-responsive genes during critical transition stages of the parasite life cycle. EMBO I. 17: 6115-6123.

Ding, A.H., Nathan, C.F., and Stuehr, D.J. 1988. Release of reactive nitrogen intermediates and reactive oxygen intermediates from mouse peritoneal macrophages. Comparison of activating cytokines and evidence for independent produc- 
tion. J. Immunol. 141: 2407-2412.

Durner, J., Wendehenne, D., and Klessig, D.F. 1998. Defense gene induction in tobacco by nitric oxide, cyclic GMP, and cyclic ADP-ribose. Proc. Natl. Acad. Sci. 95: 10328-10333.

Dushay, M.S., Asling, B., and Hultmark, D. 1996. Origins of immunity: Relish, a compound Rel-like gene in the antibacterial defense of Drosophila. Proc. Natl. Acad. Sci. 93: 10343-10347.

Ferrandon, D., Jung, A.C., Criqui, M., Lemaitre, B., UttenweilerJoseph, S., Michaut, L., Reichhart, J., and Hoffmann, J.A. 1998. A drosomycin-GFP reporter transgene reveals a local immune response in Drosophila that is not dependent on the Toll pathway. EMBO J. 17: 1217-1227.

Georgel, P., Naitza, S., Kappler, C., Ferrandon, D., Zachary, D., Swimmer, C., Kopczynski, C., Duyk, G., Reichhart, J.M., and Hoffmann, J.A. 2001. Drosophila immune deficiency (IMD) is a death domain protein that activates antibacterial defense and can promote apoptosis. Dev. Cell 1: 503-514.

Gibbs, S.M. and Truman, J.W. 1998. Nitric oxide and cyclic GMP regulate retinal patterning in the optic lobe of Drosophila. Neuron 20: 83-93.

Gottar, M., Gobert, V., Michel, T., Belvin, M., Duyk, G., Hoffmann, J.A., Ferrandon, D., and Royet, J. 2002. The Drosophila immune response against Gram-negative bacteria is mediated by a peptidoglycan recognition protein. Nature 416: 640-644.

Han, Y.S., Thompson, J., Kafatos, F.C., and Barillas-Mury, C. 2000. Molecular interactions between Anopheles stephensi midgut cells and Plasmodium berghei: The time bomb theory of ookinete invasion of mosquitoes. EMBO $J$. 19: 6030-6040.

Hedengren, M., Asling, B., Dushay, M.S., Ando, I., Ekengren, S., Wihlborg, M., and Hultmark, D. 1999. Relish, a central factor in the control of humoral but not cellular immunity in Drosophila. Mol. Cell 4: 827-837.

Hoffmann, J.A., Kafatos, F.C., Janeway, C.A., and Ezekowitz, R.A. 1999. Phylogenetic perspectives in innate immunity. Science 284: 1313-1318.

Imamura, M., Yang, J., and Yamakawa, M. 2002. cDNA cloning, characterization and gene expression of nitric oxide synthase from the silkworm, Bombyx mori. Insect. Mol. Biol. 11: 257-625.

Kim, Y.S., Han, S.J., Ryu, J.H., Choi, K.H., Hong, Y.S., Chung, Y.H., Perrot, S., Raibaud, A., Brey, P.T., and Lee, W.J. 2000. Lipopolysaccharide-activated kinase, an essential component for the induction of the antimicrobial peptide genes in Drosophila melanogaster cells. J. Biol. Chem. 275: 20712079.

Kopp, E.B. and Medzhitov, R. 1999. The Toll-receptor family and control of innate immunity. Curr. Opin. Immunol. 11: $13-18$.

Lemaitre, B., Kromer-Metzger, E., Michaut, L., Nicolas, E., Meister, M., Georgel, P., Reichhart, J.M., and Hoffmann, J.A. 1995a. A recessive mutation, immune deficiency (imd), defines two distinct control pathways in the Drosophila host defense. Proc. Nat1. Acad. Sci. 92: 9465-9469.

Lemaitre, B., Meister, M., Govind, S., Georgel, P., Steward, R., Reichhart, J.M., and Hoffmann, J.A. 1995b. Functional analysis and regulation of nuclear import of dorsal during the immune response in Drosophila. EMBO J. 14: 536-545.

Lemaitre, B., Nicolas, E., Michaut, L., Reichhart, J.M., and Hoffmann, J.A. 1996. The dorsoventral regulatory gene cassette spatzle/Toll/cactus controls the potent antifungal response in Drosophila adults. Cell 86: 973-983.

Levashina, E.A., Langley, E., Green, C., Gubb, D., Ashburner, M., Hoffmann, J.A., and Reichhart, J.M. 1999. Constitutive activation of toll-mediated antifungal defense in serpin-deficient Drosophila. Science 285: 1917-1919.

Lu, Y., Wu, L.P., and Anderson, K.V. 2001. The antibacterial arm of the drosophila innate immune response requires an IkappaB kinase. Genes \& Dev. 15: 104-110.

Luckhart, S., Vodovotz, Y., Cui, L., and Rosenberg, R. 1998. The mosquito Anopheles stephensi limits malaria parasite development with inducible synthesis of nitric oxide. Proc. Natl. Acad. Sci. 95: 5700-5705.

MacMicking, J., Xie, Q.W., and Nathan, C. 1997. Nitric oxide and macrophage function. Annu. Rev. Immunol. 15: 323350.

Manfruelli, P., Reichhart, J.M., Steward, R., Hoffmann, J.A., and Lemaitre, B. 1999. A mosaic analysis in Drosophila fat body cells of the control of antimicrobial peptide genes by the Rel proteins Dorsal and DIF. EMBO J. 18: 3380-3391.

Meng, X., Khanuja, B.S., and Ip, Y.T. 1999. Toll receptor-mediated Drosophila immune response requires Dif, an NF-кB factor. Genes \& Dev. 13: 792-797.

Michel, T., Reichhart, J.M., Hoffmann, J.A., and Royet, J. 2001. Drosophila Toll is activated by Gram-positive bacteria through a circulating peptidoglycan recognition protein. $\mathrm{Na}$ ture 414: 756-759.

Nappi, A.J., Vass, E., Frey, F., and Carton, Y. 2000. Nitric oxide involvement in Drosophila immunity. Nitric Oxide 4: 423430.

Nathan, C. and Shiloh, M.U. 2000. Reactive oxygen and nitrogen intermediates in the relationship between mammalian hosts and microbial pathogens. Proc. Natl. Acad. Sci. 97: 8841-8848.

Nicolas, E., Reichhart, J.M., Hoffmann, J.A., and Lemaitre, B. 1998. In vivo regulation of the IкB homologue cactus during the immune response of Drosophila. J. Biol. Chem. 273: 10463-10469.

Ooi, J.Y., Yagi, Y., Hu, X., and Ip, Y.T. 2002. The Drosophila Toll-9 activates a constitutive antimicrobial defense. EMBO Rep. 3: 82-87.

Qiu, P., Pan, P.C., and Govind, S. 1998. A role for the Drosophila Toll/Cactus pathway in larval hematopoiesis. Development 125: 1909-1920.

Ramet, M., Manfruelli, P., Pearson, A., Mathey-Prevot, B., and Ezekowitz, R.A. 2002. Functional genomic analysis of phagocytosis and identification of a Drosophila receptor for E. coli. Nature 416: 644-648.

Regulski, M. and Tully, T. 1995. Molecular and biochemical characterization of $d N O S$ : A Drosophila Ca2+/calmodulindependent nitric oxide synthase. Proc. Natl. Acad. Sci. 92: 9072-9076.

Reichhart, J.M., Meister, M., Dimarcq, J.L., Zachary, D., Hoffmann, D., Ruiz, C., Richards, G., and Hoffmann, J.A. 1992. Insect immunity: Developmental and inducible activity of the Drosophila diptericin promoter. EMBO J. 11: 1469-1477.

Rosetto, M., Engstrom, Y., Baldari, C.T., Telford, J.L., and Hultmark, D. 1995. Signals from the IL-1 receptor homolog, Toll, can activate an immune response in a Drosophila hemocyte cell line. Biochem. Biophys. Res. Commun. 209: 111-116.

Ruhf, M.L., Braun, A., Papoulas, O., Tamkun, J.W., Randsholt, N., and Meister, M. 2001. The domino gene of Drosophila encodes novel members of the SWI2/SNF2 family of DNAdependent ATPases, which contribute to the silencing of homeotic genes. Development 128: 1429-1441.

Silverman, N., Zhou, R., Stoven, S., Pandey, N., Hultmark, D., and Maniatis, T. 2000. A Drosophila IкB kinase complex required for Relish cleavage and antibacterial immunity. Genes \& Dev. 14: 2461-2471.

Stasiv, Y., Regulski, M., Kuzin, B., Tully, T., and Enikolopov, G. 
2001. The Drosophila nitric oxide synthase (dNOS) gene encodes a family of proteins which can modulate NOS activity by acting as dominant negative regulators. J. Biol. Chem. 28: 28.

Stoven, S., Ando, I., Kadalayil, L., Engstrom, Y., and Hultmark, D. 2000. Activation of the Drosophila NF- $\mathrm{kB}$ factor Relish by rapid endoproteolytic cleavage. EMBO Rep. 1: 347-352.

Tauszig, S., Jouanguy, E., Hoffmann, J.A., and Imler, J.L. 2000. Toll-related receptors and the control of antimicrobial peptide expression in Drosophila. Proc. Natl. Acad. Sci. 97: 10520-10525.

Tauszig-Delamasure, S., Bilak, H., Capovilla, M., Hoffmann, J.A., and Imler, J.L. 2002. Drosophila MyD88 is required for the response to fungal and Gram-positive bacterial infections. Nat. Immunol. 3: 91-97.

Tzou, P., Ohresser, S., Ferrandon, D., Capovilla, M., Reichhart, J.M., Lemaitre, B, Hoffmann, J.A., and Imler, J.L. 2000. Tissue-specific inducible expression of antimicrobial peptide genes in Drosophila surface epithelia. Immunity 13: 737 748.

Underhill, D.M. and Ozinsky, A. 2002. Toll-like receptors: Key mediators of microbe detection. Curr. Opin. Immunol. 14: 103-110.

Vidal, S., Khush, R.S., Leulier, F., Tzou, P., Nakamura, M., and Lemaitre, B. 2001. Mutations in the Drosophila dTAK1 gene reveal a conserved function for MAPKKKs in the control of rel/NF-кB-dependent innate immune responses. Genes \& Dev. 15: 1900-1912.

Wingrove, J.A. and O'Farrell, P.H. 1999. Nitric oxide contributes to behavioral, cellular, and developmental responses to low oxygen in Drosophila. Cell 98: 105-114.

Wu, L.P. and Anderson, K.V. 1998. Regulated nuclear import of Rel proteins in the Drosophila immune response. Nature 392: 93-97. 


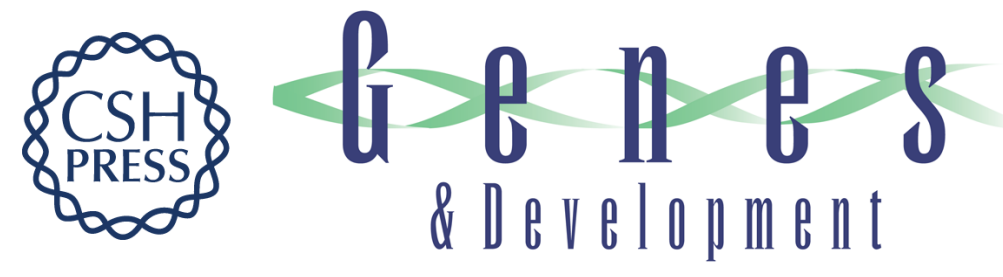

\section{Nitric oxide contributes to induction of innate immune responses to gram-negative bacteria in Drosophila}

Edan Foley and Patrick H. O'Farrell

Genes Dev. 2003, 17:

Access the most recent version at doi:10.1101/gad.1018503

References This article cites 54 articles, 28 of which can be accessed free at: http://genesdev.cshlp.org/content/17/1/115.full.htmI\#ref-list-1

License

Email Alerting

Receive free email alerts when new articles cite this article - sign up in the box at the top Service right corner of the article or click here.

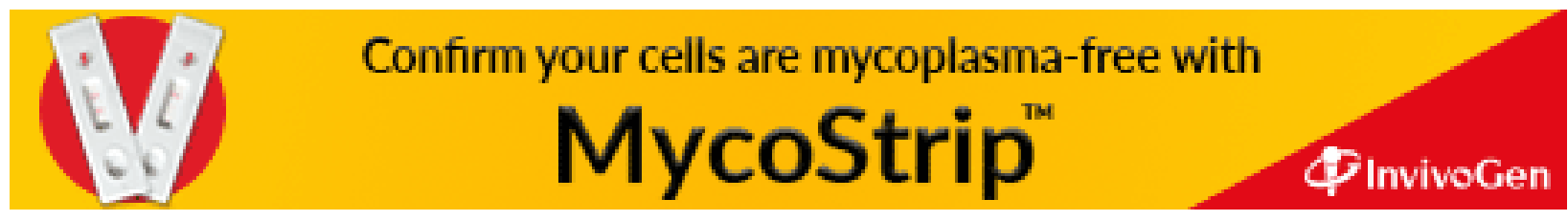

